\title{
Genome-wide identification of FoxO-dependent gene networks in skeletal muscle during C26 cancer cachexia
}

Sarah M Judge ${ }^{1}$, Chia-Ling Wu², Adam W Beharry ${ }^{1}$, Brandon M Roberts', Leonardo F Ferreira', Susan C Kandarian² and Andrew R Judge 1* $^{*}$

\begin{abstract}
Background: Evidence from cachectic cancer patients and animal models of cancer cachexia supports the involvement of Forkhead box O (FoxO) transcription factors in driving cancer-induced skeletal muscle wasting. However, the genome-wide gene networks and associated biological processes regulated by FoxO during cancer cachexia are unknown. We hypothesize that FoxO is a central upstream regulator of diverse gene networks in skeletal muscle during cancer that may act coordinately to promote the wasting phenotype.

Methods: To inhibit endogenous FoxO DNA-binding, we transduced limb and diaphragm muscles of mice with AAV9 containing the cDNA for a dominant negative (d.n.) FoxO protein (or GFP control). The d.n.FoxO construct consists of only the FoxO3a DNA-binding domain that is highly homologous to that of FoxO1 and FoxO4, and which outcompetes and blocks endogenous FoxO DNA binding. Mice were subsequently inoculated with Colon-26 (C26) cells and muscles harvested 26 days later.
\end{abstract}

Results: Blocking FoxO prevented C26-induced muscle fiber atrophy of both locomotor muscles and the diaphragm and significantly spared force deficits. This sparing of muscle size and function was associated with the differential regulation of 543 transcripts (out of 2,093) which changed in response to C26. Bioinformatics analysis of upregulated gene transcripts that required FoxO revealed enrichment of the proteasome, AP-1 and IL-6 pathways, and included several atrophy-related transcription factors, including Stat3, Fos, and Cebpb. FoxO was also necessary for the cancer-induced downregulation of several gene transcripts that were enriched for extracellular matrix and sarcomere protein-encoding genes. We validated these findings in limb muscles and the diaphragm through qRT-PCR, and further demonstrate that FoxO1 and/or FoxO3a are sufficient to increase Stat3, Fos, Cebpb, and the C/EBPß target gene, Ubr2. Analysis of the Cebpb proximal promoter revealed two bona fide FoxO binding elements, which we further establish are necessary for Cebpb promoter activation in response to IL-6, a predominant cytokine in the C26 cancer model.

Conclusions: These findings provide new evidence that FoxO-dependent transcription is a central node controlling diverse gene networks in skeletal muscle during cancer cachexia, and identifies novel candidate genes and networks for further investigation as causative factors in cancer-induced wasting.

Keywords: Muscle Atrophy, Microarray, Proteasome, Cebpb, Fos, Transcription factors, Z-disc, Extracellular matrix

\footnotetext{
* Correspondence: arjudge@phhp.ufl.edu

'Department of Physical Therapy, University of Florida, 1225 Center Drive, HPNP Building 1142, Gainesville, Florida, USA

Full list of author information is available at the end of the article
} 


\section{Background}

Cachexia is a devastating condition that affects up to $80 \%$ of patients with cancer, particularly those with cancers of the lung and upper GI tract [1]. The condition is characterized by progressive weight loss due to significant skeletal muscle wasting, in the presence or absence of adipose tissue wasting. Importantly, the muscle wasting causes significant muscle weakness that negatively affects physical function and independence, and thus quality of life. In addition, muscle and body wasting during cancer is also associated with a reduced tolerance to chemotherapy [2], increased complications from surgical and radiotherapeutic treatments [3], higher rate of metastatic disease and decreased survival [4]. Therefore, developing treatment strategies to deter cancer cachexia is critically important to enhancing the quality of life and survival of cancer patients. However, in order for this to happen, a better understanding of the mechanisms which drive muscle wasting during cancer is needed.

Skeletal muscle wasting during cancer displays marked similarities to other atrophy-inducing conditions, in that the loss of muscle mass is characterized by increased protein degradation and decreased protein synthesis [1,5]. However, recent studies demonstrate that muscle wasting during cancer is also related to disruptions in the dystrophin glycoprotein complex and muscle fiber integrity and impaired myogenic capacity [6,7], thus emphasizing the unique and complex nature of cancer cachexia. The upstream molecules implicated in driving these muscle pathologies during cancer include several pro-inflammatory cytokines that are increased in the circulation of cancer patients and tumorbearing mice $[1,8]$. Intrinsic to the muscle, mechanistic evidence demonstrates the requirement of inhibitor of kappa $B$ kinase beta (IKK $\beta$ ) activation and the subsequent degradation of the inhibitor of kappa B alpha (IkB $\alpha)[7,9,10]$ for cancer-induced muscle atrophy and myogenic impairment. Also required for the atrophy phenotype during cancer are the transcription factors, Signal Transducer and Activator of Transcription 3 (STAT3), which acts downstream of IL-6 [11,12], CCAAT/enhancer-binding protein beta $(\mathrm{C} / \mathrm{EBP} \beta)$, which is activated by $\mathrm{p} 38$ mitogen activated protein kinase (MAPK) [13], and activator protein-1 (AP-1) [14], which is activated through extracellular regulated kinase 1 and 2 (ERK 1/2) MAPK [15]. Lastly, evidence from our lab and another demonstrates that activation of Forkhead BoxO (FoxO) transcription factors also plays a causative role in cancer-induced muscle wasting $[16,17]$.

Skeletal muscle expresses three FoxO family members, including FoxO1, FoxO3 and FoxO4, with both FoxO1 and FoxO3a significantly upregulated in cachectic muscles from LLC and C26 tumor-bearing mice $[9,16]$. Moreover, FoxO1 is also upregulated in skeletal muscle of human cancer patients, and was recently identified as a cachexia-associated gene [18]. Importantly, activation of the FoxO transcription factors is both sufficient to cause muscle atrophy and necessary for muscle wasting in response to numerous catabolic conditions, including cancer cachexia associated with Lewis Lung Carcinoma [16] and Sarcoma-180 [17]. Thus, these findings from both human cancer patients and animal models of cancer cachexia strongly support the involvement of FoxO in driving the muscle atrophy process. Despite this, the genome-wide gene networks regulated by FoxO during cancer cachexia are unknown. Indeed, although the FoxO factors are well established to regulate genes involved in skeletal muscle proteolysis through the ubiquitin proteasome pathway and autophagy, due to the complex nature of cancer cachexia, we hypothesize that FoxO regulates additional gene networks which promote the wasting phenotype. Indeed, identifying the broader gene targets regulated by FoxO is an important next step which may unveil novel insight into the mechanisms which promote cancer-induced muscle wasting.

The purpose of the current study was to determine the requirement of FoxO for locomotor muscle and diaphragm muscle wasting and weakness in response to Colon-26 (C26) adenocarcinoma, and provide the first genome-wide analysis of the genes and biological networks targeted by FoxO in response to $\mathrm{C} 26$ tumor burden. We found that FoxO is necessary for C26-induced muscle wasting of both locomotor muscles and the diaphragm, and that this was associated with its regulation of genes involved in not only proteolysis, but additional atrophy-related transcriptional pathways, including the IL-6 and AP-1 pathways. In addition we also identified FoxO as a novel regulator of gene repression during cancer cachexia, with the most enriched gene networks related to the structure and functional integrity of the extracellular matrix and muscle sarcomere. The data presented in this study thus highlights novel candidate genes and biological networks that are regulated downstream of FoxO that may be further explored as causative factors in cancer-induced muscle wasting.

\section{Methods}

\section{Animals}

Male CD2F1 mice weighing $20 \mathrm{~g}$ were purchased from Charles River Laboratories (Wilmington, Massachusetts) and used for all animal experiments. Mice were maintained in a temperature and humidity-controlled facility with a 12-h light/dark cycle and water and standard diet were provided ad libitum. The University of Florida Institutional Animal Care and Use Committee approved all animal procedures.

\section{AAV vectors}

The d.n.FoxO construct used to inhibit FoxO-dependent transcription encodes for amino acids 141-266 of human FoxO3a which encompasses only the FoxO3a DNA binding domain. The amino acid sequence of the d.n.FoxO 
protein shares $100 \%$ sequence identity with mouse FoxO3a (aa140-265), 85\% sequence identity with mouse FoxO1 and $75 \%$ sequence identity with mouse FoxO4 (Figure 1), all of which share $>90 \%$ sequence conservation within this region. Since the d.n.FoxO protein lacks a transactivation domain, the d.n.FoxO blocks DNA-binding dependent transcription by the FoxO factors through outcompeting endogenous FoxO factors for binding to FoxO DNA binding elements (FBEs) in gene regulatory regions. The d.n. FoxO is fused to a DsRed protein tag to allow for quantitation of the ectopic protein and has been used and described by our lab previously $[16,19]$. The d.n.FoxO cDNA was sub-cloned into the SpeI and ClaI sites of pTR-UF12 under the control of a cytomegalovirus and chicken $\beta$ actin hybrid promoter. The pTR-UF12 shuttle vector also contains the internal ribosome entry site (IRES) that allows for the bi-cistronic expression of GFP for measurement of AAV9 transduction efficiency. The pTR-UF12-d.n.FoxO and pTR-UF12 (empty vector, ev) were packaged in rAAV9 and titered at the University of Florida Powell Gene Therapy Center Vector Core Laboratory using previously published methods [20]. The vectors were purified by iodixanol gradient centrifugation and anion-exchange chromatography, as described previously [20] and final formulations of AAV9-d.n.FoxO and AAV9-ev were provided in lactated Ringer's solution.

\section{In vivo AAV delivery}

Mice were acutely anesthetized with isoflurane gas $(5 \%$, induction; 3\% maintenance) delivered via a nose cone. For
AAV delivery to the TA and EDL, a small incision was made on the lateral side of the lower leg and the TA muscle exposed. Each vector was diluted in lactated Ringer solution such that $1 \times 10^{11}$ vector genomes (VG) were injected in $25 \mu \mathrm{l}$ along the tibia into the TA and EDL muscles, as previously described by others [21]. For targeting to the diaphragm, we performed a single intrathoracic injection of $1 \times 10^{11}$ vector genomes/mouse in $400 \mu$ lof sterile lactate Ringer's solution. This minimally invasive technique causes high and widespread transduction of the diaphragm [22,23].

\section{Plasmid DNA vectors}

Expression plasmids for the FoxO1 triple phosphorylation mutant (Addgene Plasmid 17547, deposited by Dr. Domenico Accili) and the FoxO3a triple phosphorylation mutant (Addgene Plasmid 10711, deposited by Dr. William Sellers ), have been described previously [24] and were injected and electroporated into mouse TA muscles as described by us previously [16]. The pGL4.20 luciferase reporter plasmids containing either a wildtype Cebpb promoter fragment $(-516$ to -1$)$ or a mutated version which is mutated at both FoxO binding elements (FBE1 (-234 to -225$)$ and FBE2 (-208 to -199$)$ ) were generous gifts from Dr. Akiyoshi Fukamizu, and have been described previously [25]. The pRL-TK-Renilla luciferase reporter plasmid was purchased from Promega (Madison, WI).

\section{Cancer cachexia}

Murine C26 cells were obtained from the National Cancer Institute Tumor Repository (Frederick, MD, USA) and

A

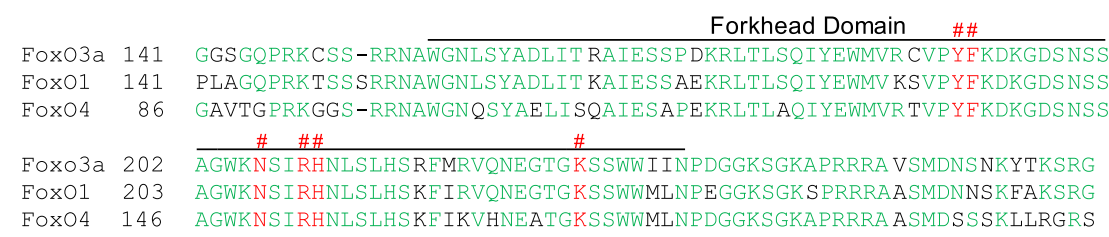

B

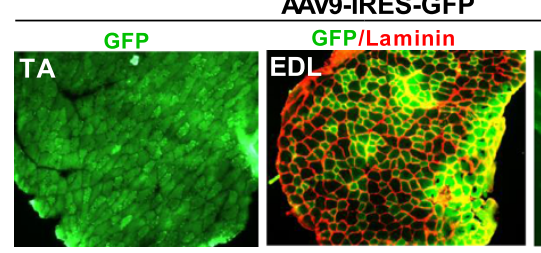

C

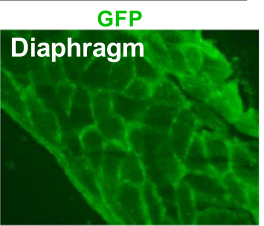

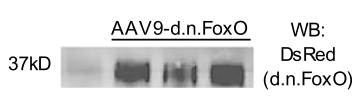

Figure 1 Transduction of locomotor muscles and the diaphragm with AAV9-d.n.FoxO. (A) Alignment of the dominant negative (d.n.) FoxO protein sequence, which includes amino acids 141-265 of mouse FoxO3a, with the corresponding mouse FoxO1 and FoxO4 amino acid sequences. FoxO1 shares $\sim 85 \%$ amino acid sequence identity and FoxO4 75\% sequence identity with the d.n.FoxO protein (shared amino acids denoted in green), all of which share $>90 \%$ sequence conservation within this region. The 6 amino acid residues involved in DNA binding of the Forkhead Domain are highlighted in red and are denoted by hash marks (\#) above the aligned sequences. (B and C) The AAV9 vectors driving expression of d.n.FoxO (or empty vector), which also contain an IRES driving the expression of GFP, were injected directly into the anterior hind limb compartment of mice to transduce the TA and EDL muscles, or injected directly into the intrathoracic cavity of mice to transduce the diaphragm. (B) Representative muscle cross-sections showing AAV9 transfection efficiency in the TA, EDL and diaphragm 26 days post-injection as visualized via direct GFP fluorescence. (C) Confirmation that the d.n.FoxO protein was also expressed in muscles transduced with AAV9-d.n. FoxO was confirmed through western blot using an antibody against DsRed, which is fused to the d.n.FoxO protein. 
cultured as described previously [26,27]. Cancer cachexia was induced in mice by injecting $5 \times 10^{5} \mathrm{C} 26$ cells (or $1 \times$ PBS as control) subcutaneously into each flank on the same day as AAV delivery. Muscles were harvested when the largest tumor diameter reached $1.5 \mathrm{~cm}$ ( $\sim 26$ days post-inoculation) when mice had lost $\sim 15 \%$ of tumor-free body weight.

\section{Histochemistry}

Transduction efficiency of AAV9 was determined in $10 \mu \mathrm{m}$ cross-sections via direct visualization of GFP fluorescence using a Leica DM5000B microscope (Leica Microsystems, Wetzlar, Germany) prior to and/or following fixation with 4\% paraformaldehyde and labeling of muscle fiber borders with Alexa Fluor-conjugated wheat germ agglutinin (Invitrogen) for $1 \mathrm{hr}$. Leica application suite, version 3.5.0 software was used to trace and measure fiber CSA as described previously [27].

\section{In vitro muscle contractile properties}

The solutions and methods used for measurements of muscle isometric function in EDL muscles were described in detail previously $[27,28]$.

\section{C2C12 cell culture and IL- 6 treatment}

Mouse $\mathrm{C} 2 \mathrm{C} 12$ skeletal myoblasts were purchased from American Type Culture Collection (Manassas, VA), and were cultured and transfected with plasmids as described previously by our lab [29]. Myotubes were treated on day 3 of differentiation with $10 \mathrm{ng} / \mathrm{mL}$ of IL- 6 for either 0 or 3 hours prior to harvest and firefly/renilla luciferase activity was measured as previously described [29].

\section{RNA isolation}

RNA was extracted from TA and diaphragm muscles using TRIzol as previously described [16]. Isolated total RNA was subsequently purified using an RNeasy Mini kit (Qiagen, Valencia, CA), according to manufacturer's instructions. The resulting quantity and purity of total RNA was tested through absorbance spectrophotometry at 230, 260 and $280 \mathrm{~nm}$, and the quality of RNA was tested on a 1\% denaturing agarose gel. Synthesis of cDNA and qRT-PCR analyses from RNA isolated from the TA and diaphragm were performed as described previously [28] using a 7300 real-time PCR system and the following primers from Applied Biosystems (Austin, TX): Fbxo30 (NM_027968.3), Fbxo31 (NM_133765.4), Bach2 (NM_001109661.1), Socs3 (NM_007707.3), Ubr2 (NM_146078.3), Psma2 (NM_ 008944.2), Ubqln1 (NM_026842.4), Fos (NM_010234.2), Cebpb (NM_009883.3), Stat3 (NM_011486.4), Col6a2 (NM_146007.2), Myoz3 (NM_133363.3), atrogin-1/MAFbx/ Fbxo32 (NM_026346.2), MuRF1/Trim63 (NM_001039048.2), Bcl3 (NM_033601.3), and Maff (NM_010755.3).

\section{Microarray}

For microarray analysis, 16 total RNA samples from two conditions (control and tumor bearing) transduced with either AAV9-ev or AAV9-d.n.FoxO (4 samples per group, 4 groups) were sent to the Boston University Medical Center Microarray Core Facility for amplification, labeling, and hybridization on the mouse Affymetrix Gene 1.0 ST array (Santa Clara, CA, USA). This microarray is designed to measure the expression of 28,132 well-annotated genes. A total of sixteen array images were acquired by GeneChip Scanner 3000 TG and the image (expression) quality was assessed by the Affymetrix Expression Console (Santa Clara, CA, USA). The Expression File Creator module of the GenePattern platform was used to generate gene expression signal values [30] and were normalized by robust multi-array analysis algorithm (RMA) [31]. Brainarray MoGene 1.0 ST custom Chip Definition File v.16 was used for probe annotation [32]. The resulting expression data for 21,225 genes were uploaded for Principle Component Analysis (PCA) on the Genepattern platform [30]. We found one outlier from the AAV9-ev C26 group using PCA and thus, this sample was removed from further analysis. The expression values were log2-transformed and preprocessed by the Pre-ProcessDataSet module of GenePattern to include genes with expression values between $1-2^{20}$, a min.fold.change $\geq 2$ and a min delta $\geq 1.2$. The last two variation filters were set to eliminate genes that showed no expression change among the 15 samples but to include genes showing changes at low expression values. In the Pre-ProcessDataSet module, min.fold. change is defined as the fold change of the 2nd highest expression value among the 15 samples compared to the 2nd lowest value among the 15 samples, whereas min delta is defined as the difference between the 2nd highest expression value and the 2 nd lowest value among the 15 samples [30]. Both .cel files and expression values were deposited into MIAME compliant NCBI Gene Expression Omnibus [33] with accession \#GSE56555. Following these filtering and preprocessing steps, 20,432 genes remained.

Differential gene expression analyses were subsequently performed using the Comparative Marker Selection module in GenePattern [30], which compares mean differences between two groups by two-way parametric t-tests. To identify differentially expressed genes in muscles from tumor-bearing mice, expression values from the AAV9-ev control group were compared to the AAV9-ev C-26 group (using $\mathrm{q} \leq 0.01$ and $-1.5 \geq$ fold change $\geq 1.5$-fold), which identified 2,194 genes. Then, to identify the direct or indirect FoxO target genes during cancer, the differentially expressed genes due to cancer were compared to expression values from the AAV9-d.n.FoxO $\mathrm{C}$-26 group $(\mathrm{q} \leq 0.01$ and $-1.5 \geq$ fold change $\geq 1.5$-fold), which identified 544 
genes. Genes which were also significantly changed by AAV9-d.n.FoxO during control conditions (AAV9-ev control vs. AAV9-d.n.FoxO control, q < 0.01), were eliminated as FoxO target genes in response to the $\mathrm{C} 26$ tumor.

Upregulated or downregulated FoxO target genes in response to the $\mathrm{C} 26$ tumor were analyzed separately for their associated functional annotations using the DAVID Bioinformatics database [34]. Enriched terms and biological networks were identified using pre-selected default annotation categories, an EASE score (a modified Fisher Exact P-value) of less than 0.05 and an enrichment score greater than 1.5. Enriched terms were clustered using the Functional Annotation Clustering tool, which groups analogous annotations together to reduce redundancy in the report. FoxO target genes were also analyzed using the Broad Institute's Molecular Signatures Database [35] to identify enriched canonical pathways and to identify the most commonly shared transcription factor binding motifs located within the $-2 \mathrm{~kb}$ to $2 \mathrm{~kb}$ cis-regulatory regions of these genes.

\section{Statistical analyses}

Methods used for statistical analysis of the microarray data are described in the results section. All other data were analyzed using ANOVA followed by Bonferroni post hoc comparisons (GraphPad Software, San Diego, CA) and significance was set at $P \leq 0.05$.

\section{Results}

FoxO is necessary for locomotor and diaphragm wasting in C26 tumor-bearing mice

Wasting of locomotor muscles is an important component of whole body weakness and fatigue in cancer patients. In addition, the diaphragm muscle also undergoes significant wasting and is believed to play a key role in respiratory complications and mortality in cancer patients. Despite this, studies aimed at understanding the mechanisms of muscle wasting during cancer have largely focused on locomotor muscles. Thus, in the current study we focused on the role of the FoxO factors in cancer-induced wasting of both locomotor muscles and the diaphragm in response to Colon-26 tumor. To inhibit endogenous FoxO1, FoxO3a and FoxO4 DNA binding-dependent transcription we transduced muscles with recombinant AAV9-d.n.FoxO (or AAV9-ev as the respective control) both of which also express GFP as a non-fusion protein to visualize transduction efficiency. Importantly, the d.n.FoxO sequence consists of only that which codes for the FoxO3a DNA binding domain, and shares $85 \%$ sequence identity with the respective DNA binding domain of FoxO1 and 75\% sequence identity with that of FoxO4 (Figure 1), all of which share $>90 \%$ sequence conservation within this region. The d.n.FoxO therefore acts through outcompeting endogenous FoxO1, FoxO3a and FoxO4 for binding to FoxO DNA binding elements, and, since it lacks a transactivation domain, blocks FoxO DNA binding-dependent transcription. To transduce locomotor muscles we performed a single intramuscular injection of AAV9 into the TA and EDL muscles of mice. To transduce the diaphragm, in a separate cohort of animals we performed a single intrathoracic injection of AAV9. Immediately following AAV9 injections, mice assigned to the tumor-bearing groups were inoculated with C26 cells, and control mice injected with $1 \times$ PBS. Muscles from control and tumor-bearing mice were harvested at tumor end point ( 26 days post C26-inoculation) when mice lose $\sim 15 \%$ of their tumor-free body mass, which has been documented by us previously [9]. Using these methods, we were able to achieve nearly 100\% AAV9 transduction efficiency of fibers in the TA muscle and the diaphragm and $\sim 75 \%$ transduction efficiency of fibers in the EDL as visualized by GFP fluorescence in muscle cross-sections (Figure 1).

As shown in Table 1 and Figure 2, presence of the C26 tumor induced significant muscle fiber atrophy in the TA, EDL, and diaphragm of mice transduced with AAV9-ev. In contrast, muscles of tumor-bearing mice transduced with AAV9-d.n.FoxO showed significant sparing of muscle fiber CSA. These data therefore demonstrate that blocking FoxO-dependent transcription is sufficient to impede C26-induced muscle wasting of both locomotor muscles and the diaphragm, which extends previous findings that FoxO is necessary for locomotor muscle wasting during LLC- and S-180-induced cancer cachexia [17]. Notably, transduction of muscles of non-tumor-bearing mice with AAV9-d.n.FoxO significantly increased fiber CSA over the 26 day period in the TA as observed previously by our lab. Increased fiber size was also observed in the EDL, but presumably due to the lower AAV9 transduction efficiency, this did not reach statistical significance $(\mathrm{p}=0.16)$ since all fibers in muscle cross-sections were measured in order to make sense of subsequent muscle force measurements in the EDL. In contrast, fiber size was not altered by AAV9-

Table 1 Effect of AAV9-d.n.FoxO on muscle fiber CSA in C26 tumor-bearing mice

\begin{tabular}{lllll}
\hline & Control AAV9-ev & Control AAV9-d.n.Foxo & C26 AAV9-ev & C26 AAV9-d.n.FoxO \\
\hline TA fiber CSA & $1489 \mu \mathrm{m}^{2} \pm 155$ & $2065^{*} \mu \mathrm{m}^{2} \pm 94$ & $1013^{*} \mu \mathrm{m}^{2} \pm 76$ & $1479^{\dagger} \mu \mathrm{m}^{2} \pm 94$ \\
EDL fiber CSA & $1518 \mu \mathrm{m}^{2} \pm 90$ & $1705 \mu \mathrm{m}^{2} \pm 75$ & $716^{*} \mu \mathrm{m}^{2} \pm 60$ & $1106^{*+} \mu \mathrm{m}^{2} \pm 57$ \\
Diaphragm fiber CSA & $1021 \mu \mathrm{m}^{2} \pm 91$ & $1024 \mu \mathrm{m}^{2} \pm 82$ & $696^{*} \mu \mathrm{m}^{2} \pm 81$ & $980^{\dagger} \mu \mathrm{m}^{2} \pm 50$ \\
\hline
\end{tabular}

${ }^{*} \mathrm{p}<0.05$ vs control AAV9-ev group, $+\mathrm{p}<0.05$ vs. AAV9-ev C26 group. Data represent mean $\pm \mathrm{SE}, \mathrm{n}=6$ mice per group. 


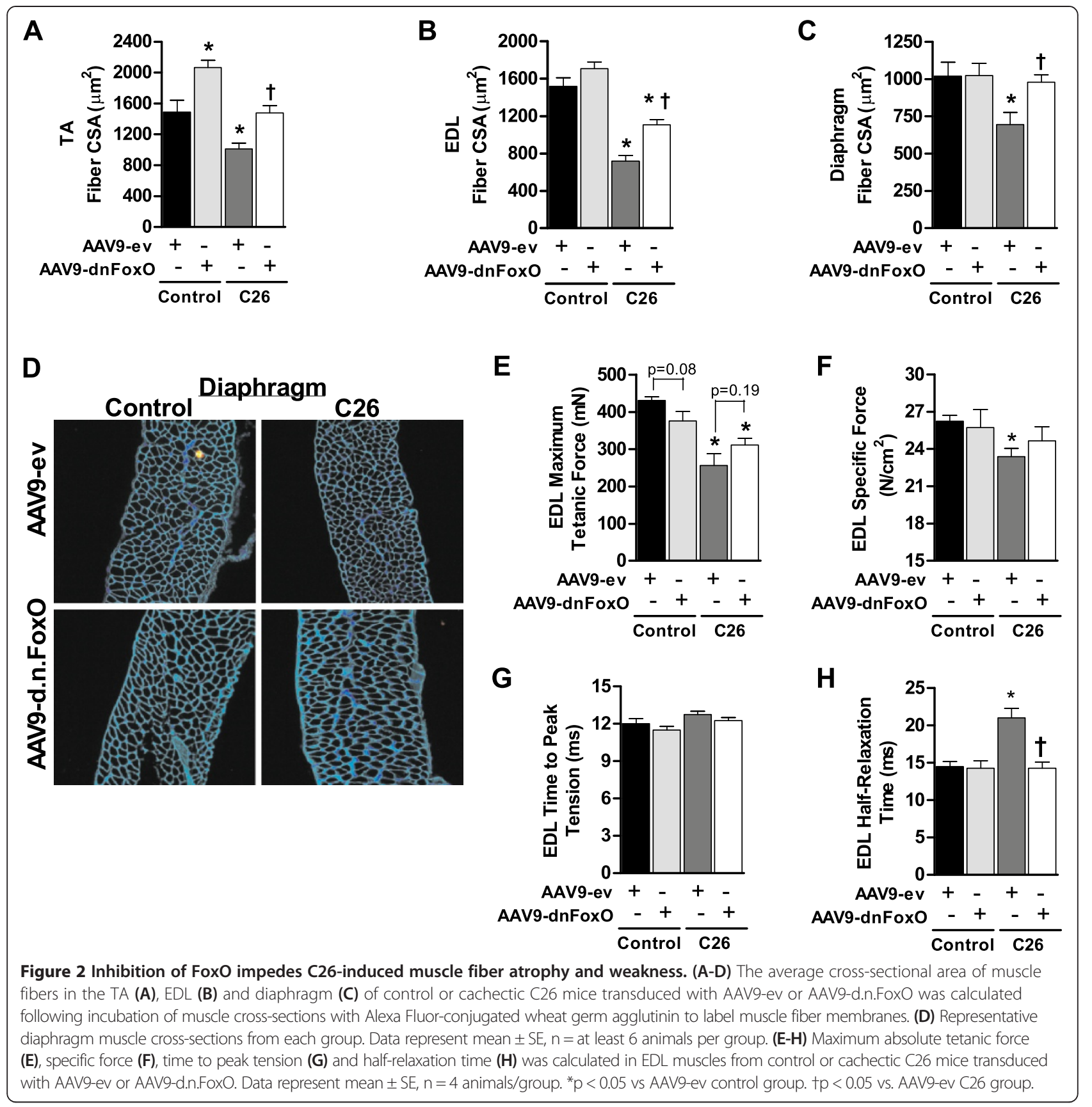

d.n.FoxO in the diaphragm of control mice despite the high transfection efficiency. Although it is unclear why blocking FoxO induced hypertrophy of limb muscles, but not the diaphragm, this differential regulation may be related to the distinct activity pattern and function of the diaphragm, which is constantly active to support breathing.

In order to determine whether the sparing of muscle fiber size in muscles of $\mathrm{C} 26$ tumor-bearing mice transduced with AAV9-d.n.FoxO carried over to sparing of muscle force deficits, we also harvested a subset of EDL muscles for measurement of in vitro contractile properties.
The rationale for choosing the EDL (over the TA and diaphragm) for force measurements is due to two main reasons: 1) the relatively small size of the EDL allows for efficient diffusion of oxygen and nutrients necessary for force measurements (which is not possible in the TA), and; 2) the EDL contains tendons on both sides which allows for both specific and maximal absolute force measurements (maximal absolute force measurements are not possible in the diaphragm). We found that EDL muscles from C26 mice transduced with AAV9-ev showed a 40\% decrease in maximum absolute force and an $11 \%$ decrease 
in specific force when compared to EDL muscles of control mice transduced with AAV9-ev, both of which were statistically significant (Figure 2E,F). In contrast, muscles from tumor-bearing mice transduced with AAV9-d.n. FoxO showed only a $28 \%$ decrease in maximum absolute force and a $6 \%$ (non-significant) decrease in specific muscle force, when compared to EDL muscles of control mice transduced with AAV9-ev. Although the attenuation of force deficits by AAV9-d.n.FoxO was not complete, these data are comparable with the effect of AAV9-d.n. FoxO on fiber size in EDL muscles of tumor-bearing mice, in which we saw only a partial sparing of fiber CSA due to the measurement of both transduced and non-transduced muscle fibers. Thus, given that only $\sim 75 \%$ of fibers were transduced with AAV9-d.n.FoxO, it seems likely that a greater attenuation of muscle weakness would have occurred had we achieved a more complete transduction of fibers within the EDL. Notably, EDL muscles from control mice transduced with AAV9-d.n.FoxO over the 26 day period showed a non-significant $(\mathrm{p}=0.09)$ decrease in maximum absolute muscle force when compared to AAV9-ev control, which suggests that chronically blocking FoxO in the absence of an atrophy stimulus may have a negative impact on force generating capacity.

Further analysis of contractile properties of the EDL demonstrated no significant differences in time to peak tension in response to the $\mathrm{C} 26$ tumor or AAV9-d.n.FoxO (Figure 2G). In contrast, half-relaxation time was significantly slowed (elevated) in response to the C26 tumor, which was completely prevented in muscles transduced with AAV9-d.n.FoxO (Figure 2H). Collectively, these data indicate that FoxO-dependent transcription is necessary for C26-induced muscle atrophy of locomotor muscles and the diaphragm, and that FoxO activation is also causative in C26-induced muscle contractile dysfunction.

\section{Microarray analysis to identify direct or indirect FoxO target genes during C26 cancer cachexia}

To comprehensively identify the gene networks changed in response to the $\mathrm{C} 26$ tumor which require FoxO-dependent transcription, we harvested TA muscles from control and cachectic C26 mice transduced with AAV9-ev or AAV9-d. n.FoxO for microarray analysis. We identified 2,194 genes that were differentially expressed between control and C26 mice injected with AAV9-ev $(-1.5 \geq$ fold change $\geq 1.5, \mathrm{q} \leq$ 0.01). Subsequent comparison of these genes between $C 26$ mice injected with either AAV9-ev or AAV9-d.n.FoxO showed that 544 genes were differentially expressed in the presence of AAV9-d.n.FoxO ( $\mathrm{q} \leq 0.01$ and fold change 21.5). Out of these genes, 1 gene (Ip6k3) was significantly changed by AAV9-d.n.FoxO during control conditions $(\mathrm{q}<0.01)$ and was thus eliminated as a downstream target of FoxO in response to the $\mathrm{C} 26$ tumor. Out of the remaining 543 genes regulated via a FoxO-dependent manner, 296 genes were upregulated in skeletal muscle due to the C26 tumor (see Additional file 1) and 247 were downregulated (see Additional file 2). To identify the broader gene networks, biological processes and canonical pathways regulated through FoxO in response to tumor burden, we functionally categorized these genes using the DAVID Bioinformatics database [34,36] and the Broad Institute Molecular Signatures Database (MSigDB) [37]. Transcripts upregulated in response to the C26 tumor were analyzed separately from transcripts downregulated in response to the $\mathrm{C} 26$ tumor.

\section{Transcription factors, including Cebpb and AP-1, are downstream targets of FoxO in cachectic muscle}

Among the 296 direct or indirect FoxO target genes upregulated in skeletal muscle of $\mathrm{C} 26$ tumor-bearing mice, the most highly enriched biological annotation clusters identified through DAVID were related to the Basic Leucine Zipper (bZIP) transcription factors, the proteasome complex, transcriptional regulation and apoptosis. Ranked in order of significance, the most highly enriched annotation term from each of the top 10 non-redundant annotation clusters identified via DAVID are shown in Figure 3B (top panel). The top 10 Broad MSigDB canonical pathways, ranked in order of significance, are also shown in Figure 3B (bottom panel), which revealed findings consistent with the DAVID analysis. Among the top 20 canonical pathways, seven were related to protein degradation, including metabolism of amino acids, the proteasome, and antigen processing: ubiquitination and proteasome degradation. An additional seven pathways were associated with inflammatory processes, including AP-1, IL-6 and apoptosis. Expression data for FoxO-regulated transcripts belonging to enriched canonical pathways of interest are shown in Table 2.

Among the FoxO-regulated genes annotated to the canonical IL-6 and/or AP-1 pathways are several bZIP transcription factors including CCAAT/enhancer-binding protein beta $(\mathrm{Cebpb})$, and factors which heterodimerize within the AP-1 transcription factor complex, including vfos FBJ murine osteosarcoma viral oncogene homolog (Fos), Fosl2, Fosb and Jun B proto-oncogene (Junb). Based on these findings we postulated that transcription factor binding motifs for C/EBP $\beta$ and AP-1, in addition to FoxO, would be enriched in the promoter regions of genes identified as indirect or direct targets of FoxO. Thus, in a separate analysis we used Broad's Gene Set Enrichment Analysis tool to analyze the $-2 \mathrm{~kb}$ to $2 \mathrm{~kb}$ cis-regulatory regions surrounding the transcriptional start site of upregulated FoxO target genes to identify the most commonly shared conserved transcription factor consensus motifs. This tool uses overlap comparison of user-provided gene lists and gene sets defined in the TRANSFAC (version 7.4) database as those which share a cis-regulatory motif 


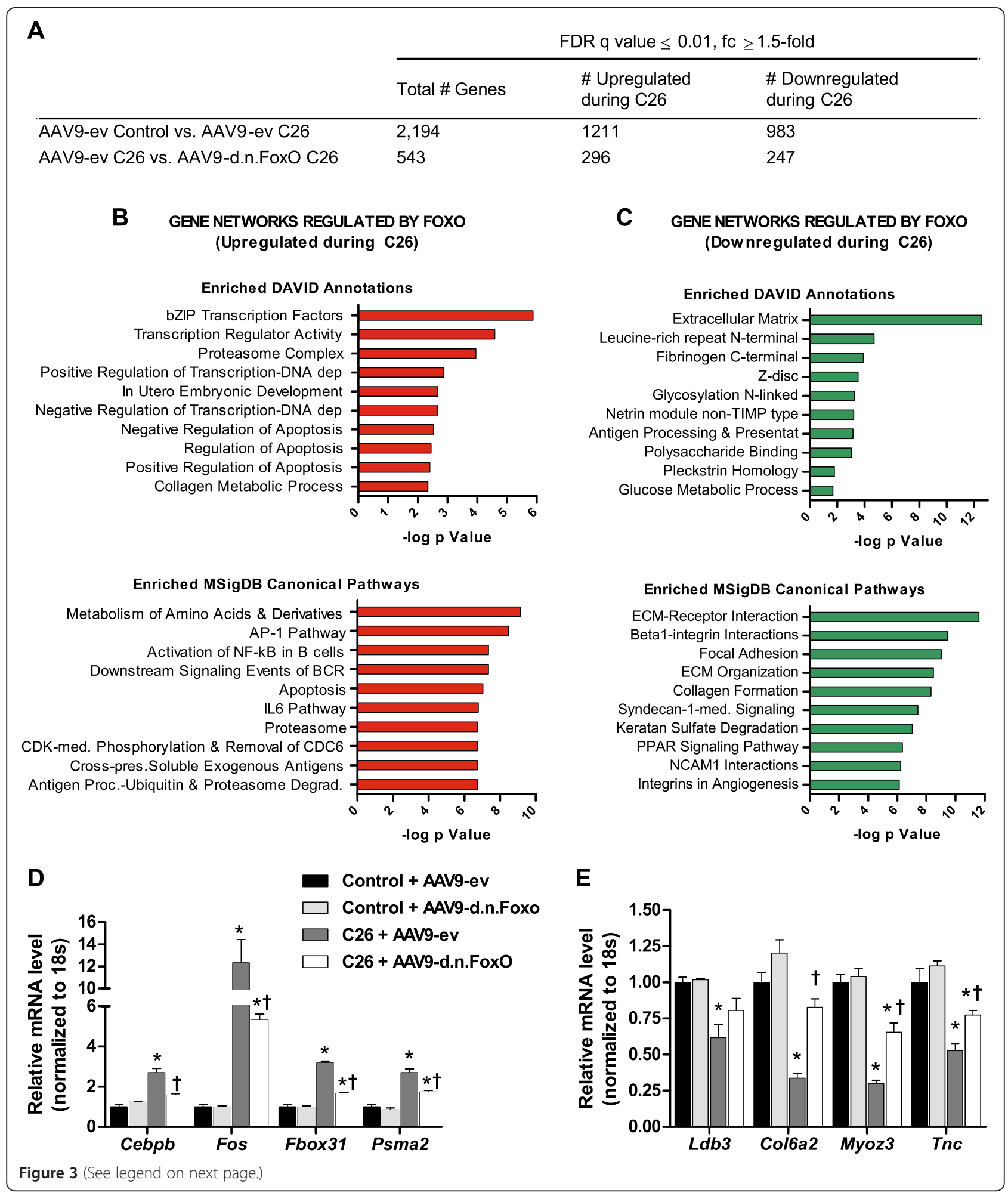




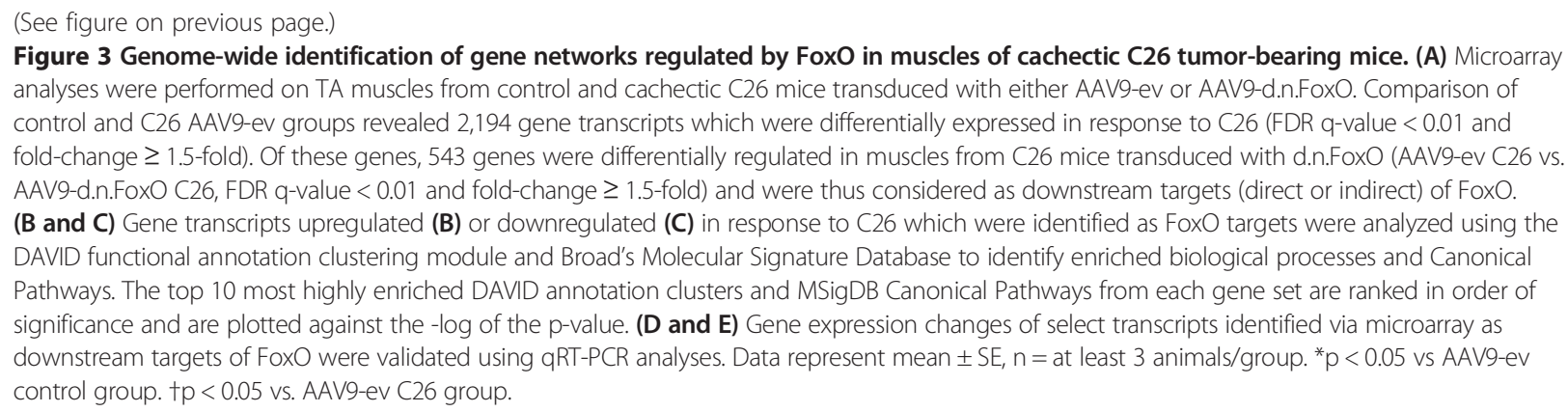

conserved across the human, mouse, rat and dog genomes. Due to the conservation of these motifs across species, these sites are more likely to reflect putative gene regulatory elements. As expected, a TTGTTT consensus motif, which is annotated to FoxO4 ( $\mathrm{q}=3.76 \mathrm{E}-13)$, and is part of the core FoxO consensus motif also regulated by FoxO1 and FoxO3a, was identified as the second most commonly shared motif (see Additional file 3: Table S1). Moreover, although less commonly shared, the complete reverse FoxO consensus motif (G/A)TAAACA (annotated to FOXF2, $q=2.08 \mathrm{E}-06$ ), which matches the FoxO DNA binding element (FBE) regulated by FoxO1 and FoxO3a in the MuRF1 promoter [38], was also significantly shared among the genes in our dataset. In addition, as hypothesized a motif corresponding to AP-1 (TGANTCA, $q=$ $8.33 \mathrm{E}-12)$ was identified as the third most commonly shared motif. Thus, although associative, these findings at least support the notion that a subset of the genes identified as downstream targets of FoxO during cancer may be related to FoxO-dependent regulation of AP-1 transcription factors. Also among the top 10 most commonly shared consensus motifs were two motifs annotated to STAT5. Although the role of STAT5 in skeletal muscle wasting is unknown, STAT3 binds to an analogous motif and was recently shown to mediate muscle atrophy in C26 tumor-bearing mice [11]. STAT3 is activated through the IL-6 Pathway, which was also among the top 10 canonical pathways regulated by FoxO in response to tumor burden. Expression data for gene transcripts regulated by FoxO that are annotated to the IL-6 pathway are shown in Table 2, which includes the bona fide STAT3 target gene, suppressor of cytokine signaling 3 (Socs3).

\section{Proteasome components enriched among FoxO targets upregulated in cachectic muscle}

Based on the analyses performed using both DAVID and the MSigDB, genes involved in proteasomal protein degradation were enriched among the gene transcripts upregulated in cachectic muscle through a FoxO-dependent manner. Expression data for FoxO-regulated genes annotated to the "Proteasome" and "Antigen processing: ubiquitination and proteasome degradation" are shown in Table 2. Included are genes that encode for various subunits of the $26 \mathrm{~S}$ proteasome, including the 20S core (Psma2 and Psma7) and the $19 \mathrm{~S}$ regulator (Psmc2, Psmc4, Psmd3 and Psmd4) which confers substrate specificity to the $26 \mathrm{~S}$ complex. Several additional genes which function as E3 ligases were also identified as downstream targets of FoxO, including F-box/WD repeat-containing protein 11 (Fbx11), Socs3, kelch-like ECH-associated protein 1 (Keap1), splA/ ryanodine receptor domain and SOCS box containing 1 (Spsb1) and F-box protein 31 (Fbxo31) (Table 2). In addition, Cathepsin-L, Gabarapl1 and Bnip3, which are known gene targets of FoxO in skeletal muscle involved in protein degradation through the lysosomal/autophagy pathway $[39,40]$, were also identified as FoxO targets during cancer. Atrogin-1/MAFbx (Fbxo32) and MuRF1 (Trim63), although slightly repressed by d.n.FoxO, did not pass the statistical criteria set for the identification of FoxO-dependent targets in limb muscles of cachectic mice (see Additional file 3: Table S2). This finding therefore suggests that in addition to FoxO, other factors likely contribute to the transcriptional upregulation of atrogin-1 and MuRF1 during cancer cachexia, which has been reported previously [13]. Alternatively, since the d.n.FoxO construct inhibits FoxO-dependent transcription through outcompeting endogenous FoxO for DNA binding, it is also possible that endogenous FoxO factors could still regulate atrogin-1 and MuRF1 transcription in the presence of d.n.FoxO through a DNA-binding independent manner.

\section{FoxO is necessary for cancer-induced downregulation of genes encoding ECM and Z-disc proteins}

In addition to the 296 FoxO target genes upregulated in skeletal muscle of $\mathrm{C} 26$ tumor-bearing mice, FoxO was also necessary for the C26-induced downregulation of 247 genes. Analysis of these genes using the Functional Clustering Tool within DAVID identified the Extracellular Matrix, Leucine-Rich Repeats, the Z-disc and Glycosylation among the most highly enriched annotations (Figure 3C, top panel). Use of the Broad Molecular Signatures Database to identify top canonical pathways from this gene set further 
Table 2 Enriched gene networks upregulated via FoxO during cancer cachexia

\begin{tabular}{|c|c|c|c|c|c|c|c|c|c|}
\hline \multicolumn{6}{|c|}{ MSigDB pathway } & \multirow[b]{2}{*}{ Gene description } & \multirow[b]{2}{*}{ Gene symbol } & \multicolumn{2}{|c|}{ C26 (Fold change) } \\
\hline 1 & 2 & 3 & 4 & 5 & 6 & & & AAV9-ev & AAV9-dnFoxO* \\
\hline$x$ & & $x$ & $x$ & & $x$ & proteasome subunit, alpha type, 7 & Psma7 & 2.40 & 1.38 \\
\hline$x$ & & $x$ & $x$ & & $x$ & proteasome subunit, alpha type, 2 & Psma2 & 2.95 & 1.73 \\
\hline$x$ & & $x$ & $x$ & & $x$ & proteasome $26 \mathrm{~S}$ subunit, ATPase, 2 & Psmc2 & 2.31 & 1.47 \\
\hline$x$ & & $x$ & $x$ & & $x$ & proteasome 265 subunit, ATPase, 4 & Psmc4 & 2.47 & 1.49 \\
\hline$x$ & & $x$ & $x$ & & $x$ & proteasome 265 subunit, non-ATPase, 3 & Psmd3 & 2.15 & 1.34 \\
\hline \multirow[t]{16}{*}{$x$} & & $x$ & $x$ & & $x$ & proteasome 265 subunit, non-ATPase, 4 & Psmd4 & 2.67 & 1.69 \\
\hline & $x$ & & & & & BCL2-like 11 (apoptosis facilitator) & $B C|2| 11$ & 3.01 & 1.82 \\
\hline & $x$ & & & $x$ & & FBJ murine osteosarcoma oncogene & Fos & 16.75 & 5.70 \\
\hline & $x$ & & & $x$ & & jun B proto-oncogene & Junb & 5.40 & 2.48 \\
\hline & $x$ & & & & & early growth response 1 & Egr1 & 4.07 & 1.95 \\
\hline & $x$ & & & & & matrix metallopeptidase 9 & Mmp9 & 2.52 & 1.57 \\
\hline & $x$ & & & & & FOS-like antigen 2 & Fosl2 & 3.50 & 2.09 \\
\hline & $x$ & & & & & angiotensinogen & Agt & 5.00 & 2.64 \\
\hline & $x$ & & & & & FBJ murine osteosarcoma oncogene B & Fosb & 3.90 & 1.43 \\
\hline & & $x$ & & & $x$ & f-box and WD-40 domain protein 11 & Fbxw11 & 1.88 & 1.15 \\
\hline & & & $x$ & $x$ & & murine thymoma viral oncogene homolog 1 & Akt1 & 2.61 & 1.54 \\
\hline & & & $x$ & $x$ & & BCL2-like 1 & $\mathrm{BCl} 2 \mathrm{|l}$ & 3.01 & 1.82 \\
\hline & & & & $x$ & $x$ & suppressor of cytokine signaling 3 & Socs3 & 6.63 & 3.08 \\
\hline & & & & $x$ & & CCAAT/enhancer binding protein beta & Cebpb & 2.94 & 1.82 \\
\hline & & & & & $x$ & kelch-like ECH-associated protein 1 & Keap1 & 1.95 & 1.25 \\
\hline & & & & & $x$ & splA/ryanodine receptor dom and SOCS box & Spsb1 & 6.36 & 3.91 \\
\hline \multicolumn{10}{|c|}{ Other genes of interest } \\
\hline & & & & & & immediate early response 5 & ler5 & 3.46 & 1.74 \\
\hline & & & & & & immediate early response 3 & ler3 & 3.02 & 1.64 \\
\hline & & & & & & ubiquilin-1 & Ubqln1 & 1.93 & 1.25 \\
\hline & & & & & & f-box protein 31 & Fbxo31 & 3.25 & 1.79 \\
\hline & & & & & & $\mathrm{BCL}$ /adenovirus $\mathrm{E} 1 \mathrm{~B}$ interacting protein 3 & Bnip3 & 2.53 & 1.51 \\
\hline & & & & & & Cathepsin L & Ctsl & 3.07 & 1.79 \\
\hline & & & & & & GABA(A) receptor-associated protein like 1 & Gabarapl1 & 4.44 & 2.52 \\
\hline & & & & & & Heme Oxygenase 1 & Hmox1 & 5.91 & 1.68 \\
\hline
\end{tabular}

Expression changes of FoxO target genes of interest belonging to enriched Molecular Signatures Database (MSigDB) Canonical Pathways are shown for C26 tumor-bearing groups transduced with AAV9-ev or AAV9-d.n.FoxO.

All data represent fold-change in response to the C26 tumor, normalized to the absolute control group (AAV9-ev control). ${ }^{*} \mathrm{q}<0.01$ vs AAV-ev C26 group. MSigDB Canonical Pathways: 1. Metabolism of Amino Acids and Derivatives, 2. AP-1, 3. Activation of NF-KB in B-cells, 4. Apoptosis, 5. IL-6, 6. Antigen Processing: Ubiquitination and Protein Degradation.

revealed ECM-receptor interactions, $\beta 1$-integrin interactions, focal adhesion, ECM organization and collagen formation as the top canonical pathways (Figure 3C, bottom panel). Expression changes of downregulated genes belonging to enriched annotation categories of interest are shown in Table 3. Several of these targets have previously been documented via microarray analysis to be downregulated in skeletal muscle of cachectic C26 tumor-bearing mice $[9,12]$, though this is the first evidence demonstrating the requirement of FoxO for their downregulation. Included among the genes downregulated in a FoxO-dependent manner were those encoding for Type I and Type VI collagens. As shown in Table 3, these collagens are part of several ECM-related pathways, and play an important role in maintaining the structural integrity of muscle during contraction. In addition, several small leucine-rich repeat proteoglycans (SLRPs) were also identified as FoxO-regulated transcripts downregulated in cachectic muscle, including Chondroadherin, Keratocan, Osteoglycin, Asporin, Fibromodulin and Lumican. While the functions of these SLRPs in skeletal muscle are not well understood, these proteins are located in the ECM where they regulate the structure 
Table 3 Enriched gene networks downregulated via FoxO during cancer cachexia

\begin{tabular}{|c|c|c|c|c|c|c|c|c|}
\hline \multicolumn{5}{|c|}{ MSigDB pathway } & \multirow[b]{2}{*}{ Gene description } & \multirow[b]{2}{*}{ Gene symbol } & \multicolumn{2}{|c|}{ C26 (Fold change) } \\
\hline 1 & 2 & 3 & 4 & 5 & & & AAV9-ev & AAV9-dnFoxO* \\
\hline$x$ & $x$ & $x$ & $x$ & $x$ & collagen, type I, alpha 2 & Col1a2 & -4.26 & -2.31 \\
\hline$x$ & $x$ & $x$ & $x$ & $x$ & collagen, type I, alpha 1 & Col1a1 & -5.20 & -2.44 \\
\hline$x$ & $x$ & $x$ & $x$ & $x$ & collagen, type VI, alpha 1 & Col6a1 & -2.64 & -1.70 \\
\hline$x$ & $x$ & $x$ & $x$ & $x$ & collagen, type VI, alpha 2 & Col6a2 & -2.69 & -1.71 \\
\hline$x$ & $x$ & $x$ & $x$ & $x$ & collagen, type Vl, alpha 3 & Col6a3 & -3.55 & -1.75 \\
\hline$x$ & $x$ & $x$ & & & thrombospondin 2 & Thbs2 & -1.62 & -1.07 \\
\hline$x$ & $x$ & $x$ & & & tenascin C (hexabrachion) & Tne & -2.43 & -1.45 \\
\hline$x$ & & $x$ & & & integrin, beta 6 & Itgb6 & -10.24 & -2.20 \\
\hline$x$ & & $x$ & & & cartilage oligomeric matrix protein & Comp & -2.48 & -1.05 \\
\hline \multirow[t]{6}{*}{$x$} & & $x$ & & & chondroadherin & Chad & -16.34 & -2.40 \\
\hline & $x$ & & & & fibrillin 1 & Fbn1 & -2.86 & -1.71 \\
\hline & & $x$ & & & Rho-associated, coiled-coil protein kinase2 & Rock2 & -1.98 & -1.16 \\
\hline & & & $x$ & $x$ & collagen, type XIV, alpha 1 (undulin) & Col14a1 & -2.08 & -1.26 \\
\hline & & & $x$ & $x$ & procollagen C-endopeptidase enhancer & Pcolce & -1.81 & -1.10 \\
\hline & & & $x$ & & matrix metallopeptidase 15 & Mmp15 & -3.12 & -2.06 \\
\hline
\end{tabular}

\section{DAVID annotation}

\begin{tabular}{|c|c|c|c|c|c|c|c|c|}
\hline 1 & 2 & 3 & 4 & 5 & & & & \\
\hline$x$ & $x$ & $x$ & & & myozenin 3 & Myoz3 & -5.53 & -1.99 \\
\hline$x$ & $x$ & $x$ & & & integrin beta 1 binding protein 2 (melusin) & Itgb1bp2 & -4.25 & -2.07 \\
\hline$x$ & $x$ & $x$ & & & junctophilin 2 & $J p h 2$ & -3.95 & -2.10 \\
\hline$x$ & $x$ & $x$ & & & synemin, intermediate filament protein & Synm & -2.61 & -1.47 \\
\hline$x$ & $x$ & $x$ & & & homer homologue 1 & Homer1 & -2.26 & -1.45 \\
\hline \multirow[t]{4}{*}{$x$} & $x$ & $x$ & & & LIM domain binding 3/Zasp/Cypher & Ldb3 & -1.84 & -1.14 \\
\hline & & & $x$ & $x$ & fibromodulin & Fmod & -3.29 & -1.29 \\
\hline & & & $x$ & $x$ & keratocan & Kera & -14.38 & -3.30 \\
\hline & & & $x$ & $x$ & lumican & Lum & -2.47 & -1.43 \\
\hline
\end{tabular}

Expression changes of FoxO target genes belonging to enriched Molecular Signatures Database (MSigDB) Canonical Pathways and DAVID functional annotation categories of interest are shown for C26 tumor-bearing groups transduced with AAV9-ev or AAV9-d.n.FoxO.

All data represent fold-change in response to the C26 tumor, normalized to the absolute control group (AAV9-ev control). ${ }^{*} \mathrm{q}<0.01$ vs AAV-ev C26 group. MSigDB Canonical Pathways: 1. Extracellular Matrix (ECM) Receptor Interactions, 2. Integrin1 Pathway, 3. Focal Adhesion, 4. ECM Organization, 5. Collagen Formation. DAVID Functional Annotations: 1. Z-disc, 2. I-band, 3. Sarcomere, 4. N-linked Glycosylation, 5. Small leucine-rich repeat proteoglycan.

and integrity of the ECM, and can also modulate growth factor interactions with their cellular receptors [41]. Also enriched among the downregulated transcripts regulated downstream of FoxO were several genes whose protein products localize to the muscle sarcomere, and in particular, the Z-disc (Table 3). Included among these are Cypher, also known as ZASP or Limb domain binding protein 3 (Ldb3), Homer1 and Myozenin 3 (Myoz3, also known as calsarcin-3), all of which play critical roles in muscle fiber integrity and function [42-44]. Although the significance of these ECM and Z-disc proteins during cancer cachexia is unknown, the integrity of the sarcomere as well as the muscle fiber membrane, which is tightly linked with the ECM, is notably disrupted in cachectic muscle $[6,45]$.

In order to gain insight into the mechanisms whereby FoxO may contribute to gene downregulation during cancer cachexia we used the Broad Institute's Gene Set Enrichment Analysis tool to identify common transcription factor consensus motifs located within the promoter regions of genes whose downregulation during cancer required FoxO. Among the top 10 most commonly shared conserved transcription factors consensus motifs were those corresponding to nuclear factor of activated T-cells (NFAT), FOXO4, myocyte-specific enhancer factor $2 \mathrm{~A}$ (MEF2A), serum response factor (SRF), and myogenic differentiation 1 (MyoD) (see Additional file 3: Table S3).

\section{qRT-PCR validation of transcripts regulated by FoxO}

To validate a subset of the transcripts identified via microarray as downstream targets of FoxO during C26, we performed qRT-PCR using cDNA generated from the same RNA samples used in the microarrays. As shown 
in Figures 3D and E, similar to our findings using microarray analysis, FoxO was necessary for the cancerinduced upregulation of Cebpb, Fos, Fbxo31 and Psma2, and was necessary for the cancer-induced downregulation of Col6a2, Myoz3 and Tnc (interaction effect, $\mathrm{p}<$ 0.05, AAV9-ev C26 vs. AAV9-d.n.FoxO C26, p<0.05). In contrast, the levels of Cypher/Ldb3 were not statistically different between the AAV9-ev C26 vs. AAV9-d.n. FoxO C26 groups.

\section{FoxO-dependent gene transcripts in the diaphragm during cancer cachexia}

As shown in Table 1, inhibition of FoxO-dependent transcription completely prevented diaphragm muscle fiber atrophy during C26-induced cachexia. Therefore, in order to determine whether FoxO regulates similar gene targets in the diaphragm as in the TA, we performed qRT-PCR analyses on diaphragm muscles. Included in these analyses were also transcripts of interest that marginally missed the fold-change and/or significance criteria to be classified as FoxO-dependent transcripts in response to cancer in the TA (see Additional file 3: Table S2). This included Stat3 and the ubiquitin E3 ligases atrogin-1/MAFbx, MuRF1, Ubr2 and Fbxo30, the latter of which was recently identified as a FoxO target gene necessary for denervation-induced atrophy [46]. As shown in Figure 4A, similar to the TA, diaphragm muscles from C26 mice showed significant increases in the gene transcripts of various genes involved in protein degradation, including Socs3, Ubqln1, Psma2, Fbxo31, Fbxo30, and Ubr2, all of which were significantly repressed in diaphragm muscles of $\mathrm{C} 26$ mice transduced with AAV9-d.n.FoxO. We also found, similar to the TA, numerous transcription factor transcripts to be upregulated in the diaphragm in response to $\mathrm{C} 26$, including the $\mathrm{b}$-ZIP transcription factors, Fos, Bach2 and Cebpb, as well as Stat3 and Bcl3 (Figure 4B), each of which were significantly repressed or abolished in diaphragm muscles transduced with AAV9-d.n.FoxO. We also found that similar to the TA muscle, in response to cancer diaphragm muscles showed a significant downregulation of the collagen transcript, Col6a2, and Myoz3, which encodes the Z-disc-associated protein Myozenin 3, and that their downregulation was blocked in muscles transduced with AAV9-d.n.FoxO (Figure 4C). In addition, the C26-induced increase in atrogin-1 (Fbxo32) and MuRF1 (Trim63) in the diaphragm were significantly repressed by d.n.FoxO. This finding is in contrast to the TA, where their repression by d.n.FoxO did not reach statistical significance. This discrepancy could be related to the increased sensitivity of qRT-PCR in comparison to microarray. We therefore validated the effect of d.n.FoxO on the C26-induced increase in atrogin-1 and MuRF1 in TA muscles via qRT-PCR, and found a greater magnitude of repression than indicated on the microarray ( $\sim 40 \%$ repression of atrogin-1, $\sim 60 \%$ repression of MuRF1), both of which reached statistical significance ( $\mathrm{p}<0.05$, data not shown). However, since the magnitude of repression by d.n.FoxO was still considerably greater for both atrogin-1 and MuRF1 in the diaphragm than in the TA, these data suggest that additional transcription factors perhaps more active in limb muscle also play a role in atrogin-1 and MuRF1 transcription during cancer cachexia, which has been reported previously [13].

\section{FoxO1 and/or FoxO3a are sufficient to upregulate Fos, Cebpb, and Stat3}

The identification of Cebpb, Fos and Stat3 as downstream targets (direct or indirect) of the FoxO factors during cancer cachexia is significant, as each of these transcription factors have been identified to regulate the muscle atrophy program. More specifically, Cebpb is necessary for muscle wasting during LLC-induced cancer cachexia [13], Stat3 for muscle wasting during $\mathrm{C} 26$ cancer cachexia [11], and Fos for denervation-induced muscle loss [47]. Moreover, the heterodimeric AP-1 transcription factor containing cJUN, (which commonly dimerizes with FOS) is also necessary for muscle wasting in AH-130 tumor-bearing rats, which further implicates FOS in cancer-induced muscle wasting [14]. To further establish the FoxO factors as upstream regulators of these atrophy-related transcription factors, we next determined whether FoxO1 and/or FoxO3a were sufficient to increase the mRNA levels of Cebpb, Fos and Stat3 in skeletal muscle. We also determined whether FoxO1 and FoxO3a were sufficient to increase the expression of Socs3 and Ubr2, which are bona fide targets of STAT3 and C/EBP $\beta$, respectively. We therefore injected and electroporated into skeletal muscle expression plasmids encoding "constitutively active" FoxO1 (FoxO1 TM) or FoxO3a (FoxO3a TM) which are mutated at the three Akt phosphorylation sites and harvested muscles 4 days later for qRT-PCR analysis. As shown in Figure 4D, FoxO1 TM significantly increased the mRNA levels of Cebpb (3.8fold) and the C/EBP $\beta$ target gene, Ubr2 (3.9-fold). On the other hand, FoxO3a TM significantly increased the mRNA levels of Stat3 (1.4-fold), Fos (4.3-fold), Cebpb (3.1-fold) and Ubr2 (3.8-fold), but not Socs3. Since both FoxO1 and FoxO3a were sufficient to increase Cebpb mRNA to similar magnitudes, we analyzed the $C e b p b$ proximal promoter and identified two potential Forkhead boxO binding elements (FBEs) within 300 base pairs upstream of the transcription start site. A literature search revealed that FoxO1 binds to these sites within the Cebpb promoter to regulate Cebpb transcription in adipocytes in response to TNF $\alpha$ [25]. We therefore determined whether these FBEs within the Cebpb promoter are also necessary for increased Cebpb transcription in response to IL-6, a predominant cytokine in the C26 model of cancer cachexia. To test this we transfected $\mathrm{C} 2 \mathrm{C} 12$ skeletal muscle cells with luciferase reporter 


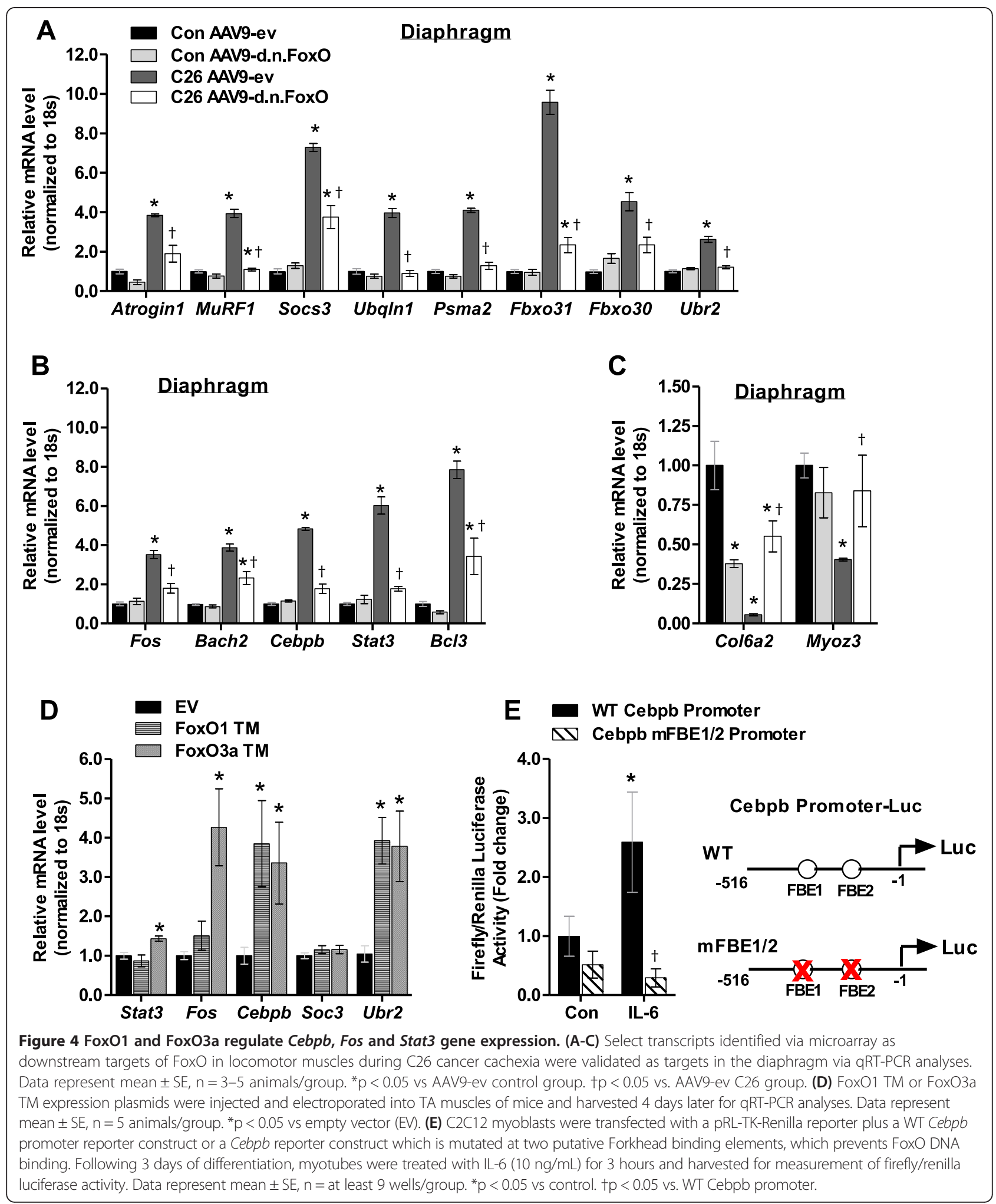

plasmids driven by either a wild type fragment of the Cebpb proximal promoter ( -516 to -1$)$ or a mutated fragment $(\mathrm{mFBE} 1 / 2)$ which contains base pair substitutions at both FBEs within the Cebpb promoter which prevent FoxO
DNA binding [25]. Following differentiation into myotubes we treated cells with IL- 6 for 3 hours and then harvested cells for measurement of luciferase activity. As shown in Figure 4E, treatment with IL-6 significantly increased 
Cebpb promoter reporter activity, which required the intact FBEs.

\section{Discussion}

Muscle wasting during cancer is a unique and multifactorial process which likely involves the differential regulation of multiple signaling pathways and downstream targets that together promote the wasting phenotype. However, the specific muscle proteins which mediate these intracellular responses to tumor burden are only beginning to be defined. In the current study we extend recent findings that the FoxO transcription factors are necessary for locomotor muscle fiber atrophy during Lewis Lung carcinoma [16] and S-180 sarcoma [17] to show that FoxO is required for skeletal muscle fiber atrophy in response to C26 adenocarcinoma in both locomotor muscles and the diaphragm, the primary muscle required for inspiration. This is significant, since diaphragm wasting during cancer may link the degree of cachexia with increased mortality. Importantly, through performing the first genome-wide microarray analysis of transcripts regulated in a FoxO-dependent manner in skeletal muscle during cancer cachexia, we reveal several gene networks changed in cachectic muscle that require FoxO. Indeed, while the FoxO factors are well known to regulate genes involved in protein degradation which was confirmed through our study, we provide new evidence that during cancer FoxO is also necessary for the gene upregulation of various atrophy-related transcription factors (and their associated transcriptional networks), including Cebpb, Stat3 and AP-1. In addition, we further demonstrate that FoxO also plays a key role in mediating the cancer-induced downregulation of various genes which function in the maintenance of muscle structural integrity. Collectively these findings highlight FoxO as a critical factor controlling diverse transcriptional networks in skeletal muscle during cancer cachexia, which provides novel insight into additional mechanisms whereby the FoxO transcription factors may orchestrate the muscle atrophy phenotype.

\section{Atrophy-related transcription factors identified as downstream targets of FoxO in response to tumor burden}

Several transcription factors were identified through microarray analysis as downstream targets of FoxO in response to tumor-burden, including the atrophy-related bZIP transcription factors, Cebpb and Fos. The identification of $C e b p b$ as a downstream target of FoxO in skeletal muscle during cancer is of particular interest, since the protein expression of $C / E B P \beta$ is increased in muscles of tumor bearing mice, and mice lacking $\mathrm{C} / \mathrm{EBP} \beta$ are resistant to LLC cancer-induced muscle wasting [13]. Moreover, we also found that both FoxO1 and FoxO3a are sufficient to increase Cebpb mRNA in skeletal muscle. Although we cannot ascertain, based on our findings, whether FoxO1 or FoxO3a directly induce Cebpb gene transcription during cancer cachexia, there are two putative FBEs located within the Cebpb proximal promoter that FoxO1 has previously been documented to bind [25]. Here we show that these FBEs are necessary for Cebpb promoter activation in skeletal muscle in response to IL-6, a predominant cytokine in the $\mathrm{C} 26$ model of cancer cachexia. Given the requirement of $\mathrm{C} / \mathrm{EBP} \beta$ for cancer-induced muscle wasting, it seems likely that FoxO-dependent upregulation of Cebpb plays a role in the muscle wasting phenotype induced by FoxO during cancer.

The significance of the immediate early gene and oncogenic transcription factor, FOS, as a FoxO target in skeletal muscle during cancer cachexia is currently unknown. However, Fos was the third most highly upregulated FoxO target gene in response to C26 (16-fold), which is in alignment with a previous microarray study showing upregulation of Fos in muscles from both moderately and severely cachectic C26 tumor-bearing mice [12]. Importantly, Fos is also upregulated in skeletal muscle following denervation and knockdown of Fos prevents the associated muscle atrophy, thus highlighting its role in the atrophy program [47]. Despite the unknown role of FOS in cancer-induced wasting, FOS heterodimerizes with c-JUN within the AP-1 transcription factor complex, which has been established as a factor required for muscle wasting in AH-130 tumor-bearing rats [14]. Since the AP-1 pathway was identified as a top canonical pathway regulated by FoxO in cachectic muscle, it seems logical that FoxOdependent induction of Fos could play a role in the enrichment of this pathway. Notably, in addition to Fos we also found that FoxO was necessary for the C26-induced increase in other early response genes related to the AP-1 pathway, including immediate early response 5 (Ier5), and early growth response 1 (Egr-1). Importantly, both Egr-1 and Ier5 were recently identified as cachexia-associated genes upregulated in muscles of pancreatic cancer patients together with Foxo1 [18]. Erg-1 was increased greater than 60-fold in patients exhibiting cachexia compared to noncachectic cancer patients, and is considered a master regulator of inflammatory response. To our knowledge these findings are the first to link FoxO to the regulation the AP-1 pathway during cancer cachexia which represents a potentially novel role for FoxO in mediating the wasting phenotype during cancer.

\section{FoxO regulates proteolytic genes in skeletal muscle in response to tumor burden}

Many of the upregulated genes identified as downstream targets of FoxO in muscles of $\mathrm{C} 26$ tumor-bearing mice are involved in protein degradation, and are coordinately upregulated during multiple wasting conditions [48-50]. 
Included among these were various proteasome subunits and Ubiquilin-1 (Ubqln1) which encodes for an ubiquitinlike (UBL) protein which physically interacts with both proteasomes and ubiquitin ligases and is involved in protein degradation [51]. In addition, several genes whose protein products function in ubiquitin E3 ligase complexes were also identified as downstream targets of FoxO, including Socs3, Fbxw11, Keap1, Spsb1 and Fbxo31. While little is known about the substrates and functions of these E3 ligases in skeletal muscle, Fbxw11 (also known as $\beta$-TrCP2) plays a role in targeting ІкB $\alpha$ for ubiquitindependent degradation [52]. This finding is intriguing, since the degradation of IKB $\alpha$ is necessary for muscle wasting during cancer $[7,9,10]$. Importantly, FoxO is wellestablished to regulate proteolysis during wasting conditions [39,40,53], and we confirmed several known FoxO targets involved in proteolysis as dependent targets of FoxO during cancer. Included among these were autophagyrelated target genes (Bnip3, Cathepsin-L and Gabarapl1), as well as Fbxo30/MUSA1, which was recently reported as a novel FoxO target gene which encodes an ubiquitin E3 ligase that is required for denervation-induced muscle loss [46]. Thus, based on these findings, it is reasonable to speculate that FoxO-dependent upregulation of these genes involved in proteolysis through both the ubiquitin proteasome pathway and the lysosomal/autophagy pathway likely plays an important role in cancer-induced wasting.

\section{FoxO is necessary for ECM and sarcomere gene downregulation in response to tumor burden}

Unexpectedly, nearly half of the genes regulated by FoxO during cancer were downregulated in response to the $\mathrm{C} 26$ tumor. While FoxO could mediate gene repression through direct binding to gene promoters, as has been documented previously [54-56], FoxO may also regulate gene repression through indirect mechanisms. This is suggested by the promoter analyses performed on downregulated target genes which identified enrichment of conserved binding motifs for not only FoxO, but several transcription factors that regulate muscle gene products, including NFAT, MEF2, and MyoD. Thus, FoxO could indirectly contribute to gene repression through inhibiting the activity of these and other transcription factors. One mechanism whereby FoxO could mediate this is through increasing the expression of transcriptional repressors and co-repressors. In fact, inhibitor of DNA-binding/differentiation proteins 1 (Id1) and 3 (Id3), which act as transcriptional repressors of myogenic basic helix-loop-helix (bHLH) proteins such as MyoD [57], were both identified as downstream targets of FoxO increased in cachectic muscle. The direct and indirect mechanisms whereby FoxO contributes to gene repression during cancer thus warrant further investigation, and will be important in moving this work forward.
A significant number of the downregulated FoxO targets during cancer encode for proteins that localize to the ECM and the Z-disc of the muscle sarcomere. Despite the relatively long half-life of typical structural proteins, prolonged downregulation of these transcripts over several weeks or even months during the progression of cancer should logically be sufficient to decrease protein expression. Provided this holds true for even a small subset of the downregulated targets, the impact of their downregulation could be highly significant to the progression of cancer-induced wasting and weakness. Indeed, many of the target genes downregulated via FoxO play critical roles in maintaining muscle integrity, fiber size and contractile function. Just one example, the extracellular matrix protein, Tenascin $C$ (Tnc), is increased in response to muscle damage, exerts anabolic and proliferative effects on interstitial and myogenic cells, and intriguingly, its genetic ablation is sufficient to decrease muscle mass, cause selective atrophy of type II muscle fibers, and slow muscle contractile properties [58]. Since these phenotypes are also characteristic of muscle wasting during cancer, it is intriguing to speculate that the downregulation of Tnc could play a role in these pathologies.

Strikingly, many of the ECM-related FoxO target genes downregulated in cachectic muscle of C26 tumor-bearing mice, including the collagen VI encoding genes, are predominately expressed and secreted by interstitial fibroblasts [59]. Indeed, fibroblasts and other stromal cells are the primary cells responsible for synthesizing the ECM and the collagen network. Although the effect of cancer on the muscle ECM and collagen network are not well defined, fibroblast activation protein- $\alpha$ (FAP)-positive stromal cells are significantly reduced in skeletal muscles of C26 tumor-bearing mice and depletion of these cells is sufficient to induce muscle fiber atrophy [60]. Moreover, collagen VI produced from muscle fibroblasts is critical to the structural integrity and function of the muscle and is also an important component of the satellite cell niche, that is necessary for satellite cell self-renewal and muscle regeneration [61]. In addition to repressing the expression of several collagen transcripts, we also found that FoxO was necessary for the coordinate increase in the gene expression of matrix metalloproteinase $8(\mathrm{Mmp} 8)$ and $M m p 9$ in muscles of cachectic tumorbearing mice (Table 2 and Additional file 3: Table S1), which participate in the degradation of Type I, II and III collagens and Type IV collagens, respectively. Therefore, determining whether the ECM and collagen network are disrupted in skeletal muscle during cancer cachexia, and whether this is mediated through a FoxO-dependent manner, warrants further investigation. Moreover, since we know that fibroblasts are the predominant source of collagen and other ECM components identified as downstream targets of FoxO during cancer, determining the role of the FoxO factors in regulating muscle fibroblasts as it relates to cancer cachexia also warrants further study. 


\section{Conclusions}

In summary, the current study identifies FoxO as a critical factor required for muscle wasting in locomotor muscles and the diaphragm in response to $\mathrm{C} 26$ cancer cachexia. Moreover, these findings provide new evidence that FoxO-dependent transcription is a central node controlling diverse gene networks in skeletal muscle during cancer cachexia which may act coordinately to regulate the atrophy phenotype. Indeed, our findings indicate that FoxO regulates not only genes involved in proteolysis, but also acts as an upstream regulator of several transcriptional networks, including C/EBP $\beta$ and the AP1 and IL-6 pathways. Intriguingly, our findings also indicate that FoxO plays a critical role in the cancer-induced downregulation of genes involved in ECM and sarcomere structure, which suggests an entirely novel role for FoxO in regulating muscle integrity during cancer. The data presented in this study thus highlights several novel candidate genes and biological networks that are targeted by FoxO that may be further explored as causative factors in cancer-induced muscle wasting.

\section{Additional files}

\section{Additional file 1: List of FoxO target genes upregulated in skeleta muscle during C26 cancer cachexia.}

Additional file 2: List of FoxO target genes downregulated in skeletal muscle during C26 cancer cachexia.

Additional file 3: Tables S1, S2 and S3. Enriched transcription factor binding motifs in promoter regions of FoxO target genes upregulated (Table S1) and downregulated (Table S3) during C26 cancer cachexia. Table S2. Expression data for additional gene transcripts of interest.

\section{Abbreviations}

AAV9: Adeno-associated virus serotype 9; bZIP: Basic Leucine Zipper; C26: Colon 26; C/EBPß: CCAAT/enhancer-binding protein beta; CSA: Cross-sectional area; DAVID: Database for Annotation, Visualization and Integrated Discovery; $\mathrm{d}$. n.: Dominant negative; ERK 1/2: Extracellular regulated kinase 1 and 2; FoxO: Forkhead box O; GFP: Green fluorescent protein; IRES: Internal ribosome entry site; LLC: Lewis lung carcinoma; MSigDB: Molecular Signatures Database; NF-kB: nuclear factor kappa B; p38 MAPK: p38 mitogen-activated protein kinase; TA: Tibialis anterior; WT: Wild type.
\end{abstract}

\section{Competing interests}

The authors declare that they have no competing interests.

\section{Authors' contributions}

ARJ and SMJ conceived and designed the study. AWB and BMR performed the animal experiments and processed tissue for biochemical and histological analyses. LFF performed muscle function experiments and analyses. CW performed pre-processing and statistical analyses of microarray data with contributions from SCK, SMJ and CW performed bioinformatics analyses. ARJ LFF and SCK contributed reagents/materials/analysis tools. SMJ wrote the paper with contributions from ARJ. All authors edited and approved the final manuscript.

\section{Acknowledgements}

This work was supported by U.S. National Institute of Arthritis and Musculoskeletal and Skin Diseases grants R01AR060209 (to A.R.J.), R00HL098453 (to L.F.F.) and R01AR060217 (to S.C.K.).

\section{Author details}

'Department of Physical Therapy, University of Florida, 1225 Center Drive, HPNP Building 1142, Gainesville, Florida, USA. ²Department of Health Sciences, Boston University, Boston, Massachusetts, USA. ${ }^{3}$ Department of Applied Physiology and Kinesiology, University of Florida, Gainesville, Florida, USA.

Received: 22 September 2014 Accepted: 11 December 2014 Published: 24 December 2014

\section{References}

1. Tisdale MJ. Mechanisms of cancer cachexia. Physiol Rev. 2009;89(2):381-410.

2. Andreyev HJ, Norman AR, Oates J, Cunningham D. Why do patients with weight loss have a worse outcome when undergoing chemotherapy for gastrointestinal malignancies? Eur J Cancer. 1998;34(4):503-9.

3. Donohoe $\mathrm{CL}$, Ryan AM, Reynolds JV. Cancer cachexia: mechanisms and clinical implications. Gastroenterol Res Pract. 2011;2011:601434.

4. Bachmann J, Heiligensetzer M, Krakowski-Roosen H, Buchler MW, Friess H, Martignoni ME. Cachexia worsens prognosis in patients with resectable pancreatic cancer. J Gastrointest Surg. 2008;12(7):1193-201.

5. Smith $\mathrm{KL}$, Tisdale MJ. Increased protein degradation and decreased protein synthesis in skeletal muscle during cancer cachexia. Br J Cancer. 1993;67(4):680-5.

6. Acharyya S, Butchbach ME, Sahenk Z, Wang H, Saji M, Carathers M, et al. Dystrophin glycoprotein complex dysfunction: a regulatory link between muscular dystrophy and cancer cachexia. Cancer Cell. 2005;8(5):421-32.

7. He WA, Berardi E, Cardillo VM, Acharyya S, Aulino P, Thomas-Ahner J, et al. NF-kappaB-mediated Pax7 dysregulation in the muscle microenvironment promotes cancer cachexia. J Clin Invest. 2013;123(11):4821-35.

8. Onesti JK, Guttridge DC. Inflammation based regulation of cancer Cachexia. BioMed Res Int. 2014:2014:168407.

9. Cornwell EW, Mirbod A, Wu CL, Kandarian SC, Jackman RW. C26 cancer-induced muscle wasting is IKKbeta-dependent and NF-kappaB-independent. PLoS One. 2014;9(1):e87776.

10. Cai D, Frantz JD, Tawa Jr NE, Melendez PA, Oh BC, Lidov HG, et al. IKKbeta/NF-kappaB activation causes severe muscle wasting in mice. Cell. 2004;119(2):285-98.

11. Bonetto A, Aydogdu T, Jin X, Zhang Z, Zhan R, Puzis L, et al. JAK/STAT3 pathway inhibition blocks skeletal muscle wasting downstream of IL-6 and in experimental cancer cachexia. Am J Physiol Endocrinol Metab. 2012;303 (3):E410-21

12. Bonetto A, Aydogdu T, Kunzevitzky N, Guttridge DC, Khuri S, Koniaris LG, et al. STAT3 activation in skeletal muscle links muscle wasting and the acute phase response in cancer cachexia. PLoS One. 2011;6(7):e22538.

13. Zhang G, Jin B, Li YP. C/EBPbeta mediates tumour-induced ubiquitin ligase atrogin1/MAFbx upregulation and muscle wasting. EMBO J. 2011:30(20):4323-35

14. Moore-Carrasco R, Garcia-Martinez C, Busquets S, Ametller E, Barreiro E, Lopez-Soriano FJ, et al. The AP-1/CJUN signaling cascade is involved in muscle differentiation: implications in muscle wasting during cancer cachexia. FEBS Lett. 2006:580(2):691-6.

15. Penna F, Costamagna D, Fanzani A, Bonelli G, Baccino FM, Costelli P. Muscle wasting and impaired myogenesis in tumor bearing mice are prevented by ERK inhibition. PLoS One. 2010;5(10):e13604.

16. Reed SA, Sandesara PB, Senf SM, Judge AR. Inhibition of FoxO transcriptional activity prevents muscle fiber atrophy during cachexia and induces hypertrophy. FASEB J. 2012;26(3):987-1000.

17. Liu CM, Yang Z, Liu CW, Wang R, Tien P, Dale $R$, et al. Effect of RNA oligonucleotide targeting Foxo-1 on muscle growth in normal and cancer cachexia mice. Cancer Gene Ther. 2007;14(12):945-52.

18. Skorokhod A, Bachmann J, Giese NA, Martignoni ME, Krakowski-Roosen H. Real-imaging CDNA-AFLP transcript profiling of pancreatic cancer patients: Egr-1 as a potential key regulator of muscle cachexia. BMC Cancer. 2012;12:265.

19. Senf SM, Dodd SL, Judge AR. FOXO signaling is required for disuse muscle atrophy and is directly regulated by Hsp70. Am J Physiol Cell Physiol. 2010;298(1):C38-45.

20. Zolotukhin S, Potter M, Zolotukhin I, Sakai Y, Loiler S, Fraites Jr TJ, et al. Production and purification of serotype 1,2, and 5 recombinant adeno-associated viral vectors. Methods. 2002;28(2):158-67.

21. Pacak CA, Walter GA, Gaidosh G, Bryant N, Lewis MA, Germain S, et al. Long-term skeletal muscle protection after gene transfer in a mouse model of LGMD-2D. Mol Ther. 2007;15(10):1775-81. 
22. Falk DJ, Mah CS, Soustek MS, Lee KZ, Elmallah MK, Cloutier DA, et al. Intrapleural administration of AAV9 improves neural and cardiorespiratory function in Pompe disease. Mol Ther. 2013;21(9):1661-7.

23. Blankinship MJ, Gregorevic P, Allen JM, Harper SQ, Harper H, Halbert CL, et al. Efficient transduction of skeletal muscle using vectors based on adeno-associated virus serotype 6. Mol Ther. 2004:10(4):671-8.

24. Ramaswamy S, Nakamura N, Sansal I, Bergeron L, Sellers WR. A novel mechanism of gene regulation and tumor suppression by the transcription factor FKHR. Cancer Cell. 2002;2(1):81-91.

25. Ito Y, Daitoku H, Fukamizu A. Foxo1 increases pro-inflammatory gene expression by inducing C/EBPbeta in TNF-alpha-treated adipocytes. Biochem Biophys Res Commun. 2009;378(2):290-5.

26. Roberts BM, Ahn B, Smuder AJ, Al-Rajhi M, Gill LC, Beharry AW, et al. Diaphragm and ventilatory dysfunction during cancer cachexia. FASEB J. 2013;27(7):2600-10.

27. Roberts BM, Frye GS, Ahn B, Ferreira LF, Judge AR. Cancer cachexia decreases specific force and accelerates fatigue in limb muscle. Biochem Biophys Res Commun. 2013;435(3):488-92.

28. Beharry AW, Sandesara PB, Roberts BM, Ferreira LF, Senf SM, Judge AR. HDAC1 activates FoxO and is both sufficient and required for skeletal muscle atrophy. J Cell Sci. 2014;127(Pt 7):1441-53.

29. Senf SM, Sandesara PB, Reed SA, Judge AR. p300 Acetyltransferase activity differentially regulates the localization and activity of the FOXO homologues in skeletal muscle. Am J Physiol Cell Physiol. 2011;300(6):C1490-501.

30. Kuehn H, Liberzon A, Reich M, Mesirov JP. Using GenePattern for gene expression analysis. Curr Protoc Bioinformatics. 2008;Chapter 7:Unit 712.

31. Bolstad BM, Irizarry RA, Astrand M, Speed TP. A comparison of normalization methods for high density oligonucleotide array data based on variance and bias. Bioinformatics. 2003;19(2):185-93.

32. Dai M, Wang P, Boyd AD, Kostov G, Athey B, Jones EG, et al. Evolving gene/ transcript definitions significantly alter the interpretation of GeneChip data. Nucleic Acids Res. 2005;33(20):e175

33. NCBI Gene Expression Omnibus. http://www.ncbi.nlm.nih.gov/geo/query/ acc.cgi?token=cbafomemplujfgp\&acc=GSE56555

34. da Huang W, Sherman BT, Lempicki RA. Systematic and integrative analysis of large gene lists using DAVID bioinformatics resources. Nat Protoc. 2009;4 (1):44-57.

35. Broad Institute Molecular Signatures Database. http://www.broadinstitute. org/gsea/msigdb/index.jsp.

36. da Huang W, Sherman BT, Lempicki RA. Bioinformatics enrichment tools: paths toward the comprehensive functional analysis of large gene lists. Nucleic Acids Res. 2009:37(1):1-13

37. Subramanian A, Tamayo P, Mootha VK, Mukherjee S, Ebert BL, Gillette MA, et al. Gene set enrichment analysis: a knowledge-based approach for interpreting genome-wide expression profiles. Proc Natl Acad Sci U S A. 2005;102(43):15545-50

38. Waddell DS, Baehr LM, van den Brandt J, Johnsen SA, Reichardt HM, Furlow JD, et al. The glucocorticoid receptor and FOXO1 synergistically activate the skeletal muscle atrophy-associated MuRF1 gene. Am J Physiol Endocrinol Metab. 2008;295(4):E785-97.

39. Mammucari C, Milan G, Romanello V, Masiero E, Rudolf R, Del Piccolo P, et al. FoxO3 controls autophagy in skeletal muscle in vivo. Cell Metab. 2007;6(6):458-71.

40. Zhao J, Brault JJ, Schild A, Cao P, Sandri M, Schiaffino S, et al. FoxO3 coordinately activates protein degradation by the autophagic/lysosomal and proteasomal pathways in atrophying muscle cells. Cell Metab. 2007;6(6):472-83.

41. Dellett M, Hu W, Papadaki V, Ohnuma S. Small leucine rich proteoglycan family regulates multiple signalling pathways in neural development and maintenance. Dev Growth Differ. 2012;54(3):327-40.

42. Stiber JA, Zhang ZS, Burch J, Eu JP, Zhang S, Truskey GA, et al. Mice lacking Homer 1 exhibit a skeletal myopathy characterized by abnormal transient receptor potential channel activity. Mol Cell Biol. 2008;28(8):2637-47.

43. Frey N, Olson EN. Calsarcin-3, a novel skeletal muscle-specific member of the calsarcin family, interacts with multiple Z-disc proteins. J Biol Chem. 2002;277(16):13998-4004.

44. Zhou Q, Chu PH, Huang C, Cheng CF, Martone ME, Knoll G, et al. Ablation of Cypher, a PDZ-LIM domain Z-line protein, causes a severe form of congenital myopathy. J Cell Biol. 2001;155(4):605-12.

45. Shum AM, Mahendradatta T, Taylor RJ, Painter AB, Moore MM, Tsoli M, et al. Disruption of MEF2C signaling and loss of sarcomeric and mitochondrial integrity in cancer-induced skeletal muscle wasting. Aging. 2012;4(2):133-43.
46. Sartori R, Schirwis E, Blaauw B, Bortolanza S, Zhao J, Enzo E, et al. BMP signaling controls muscle mass. Nat Genet. 2013;45(11):1309-18.

47. Choi MC, Cohen TJ, Barrientos T, Wang B, Li M, Simmons BJ, et al. A direct HDAC4-MAP kinase crosstalk activates muscle atrophy program. Mol Cell. 2012;47(1):122-32.

48. Sacheck JM, Hyatt JP, Raffaello A, Jagoe RT, Roy RR, Edgerton VR, et al. Rapid disuse and denervation atrophy involve transcriptional changes similar to those of muscle wasting during systemic diseases. FASEB J. 2007;21(1):140-55.

49. Lecker SH, Jagoe RT, Gilbert A, Gomes M, Baracos V, Bailey J, et al. Multiple types of skeletal muscle atrophy involve a common program of changes in gene expression. FASEB J. 2004;18(1):39-51.

50. Stevenson EJ, Giresi PG, Koncarevic A, Kandarian SC. Global analysis of gene expression patterns during disuse atrophy in rat skeletal muscle. J Physiol. 2003;551(Pt 1):33-48.

51. Rothenberg C, Monteiro MJ. Ubiquilin at a crossroads in protein degradation pathways. Autophagy. 2010;6(7):979-80.

52. Suzuki H, Chiba T, Kobayashi M, Takeuchi M, Suzuki T, Ichiyama A, et al. IkappaBalpha ubiquitination is catalyzed by an SCF-like complex containing Skp1, cullin-1, and two F-box/WD40-repeat proteins, betaTrCP1 and betaTrCP2. Biochem Biophys Res Commun. 1999;256(1):127-32.

53. Sandri M, Sandri C, Gilbert A, Skurk C, Calabria E, Picard A, et al. Foxo transcription factors induce the atrophy-related ubiquitin ligase atroginand cause skeletal muscle atrophy. Cell. 2004;117(3):399-412.

54. Potente M, Urbich C, Sasaki K, Hofmann WK, Heeschen C, Aicher A, et al. Involvement of Foxo transcription factors in angiogenesis and postnatal neovascularization. J Clin Invest. 2005;115(9):2382-92.

55. Hernandez-Ochoa EO, Schachter TN, Schneider MF. Elevated nuclear Foxo1 suppresses excitability of skeletal muscle fibers. Am J Physiol Cell Physiol. 2013;305(6):C643-53

56. Birkenkamp KU, Essafi A, van der Vos KE, da Costa M, Hui RC, Holstege F, et al. FOXO3a induces differentiation of Bcr-Abl-transformed cells through transcriptional down-regulation of Id1. J Biol Chem. 2007;282(4):2211-20.

57. Jen $Y$, Weintraub $H$, Benezra R. Overexpression of Id protein inhibits the muscle differentiation program: in vivo association of Id with E2A proteins. Genes Dev. 1992;6(8):1466-79.

58. Fluck M, Mund SI, Schittny JC, Klossner S, Durieux AC, Giraud MN Mechano-regulated tenascin-C orchestrates muscle repair. Proc Natl Acad Sci U S A. 2008;105(36):13662-7.

59. Zou Y, Zhang RZ, Sabatelli P, Chu ML, Bonnemann CG. Muscle interstitial fibroblasts are the main source of collagen VI synthesis in skeletal muscle: implications for congenital muscular dystrophy types Ullrich and Bethlem. J Neuropathol Exp Neurol. 2008;67(2):144-54.

60. Roberts EW, Deonarine A, Jones JO, Denton AE, Feig C, Lyons SK, et al. Depletion of stromal cells expressing fibroblast activation protein-alpha from skeletal muscle and bone marrow results in cachexia and anemia. J Exp Med. 2013;210(6):1137-51.

61. Urciuolo A, Quarta M, Morbidoni V, Gattazzo F, Molon S, Grumati P, et al. Collagen VI regulates satellite cell self-renewal and muscle regeneration. Nat Commun. 2013:4:1964

\section{doi:10.1186/1471-2407-14-997}

Cite this article as: Judge et al:: Genome-wide identification of FoxOdependent gene networks in skeletal muscle during C26 cancer cachexia. BMC Cancer 2014 14:997.

\section{Submit your next manuscript to BioMed Central and take full advantage of:}

- Convenient online submission

- Thorough peer review

- No space constraints or color figure charges

- Immediate publication on acceptance

- Inclusion in PubMed, CAS, Scopus and Google Scholar

- Research which is freely available for redistribution 